\title{
Metastatic gastric cancer - focus on targeted therapies
}

This article was published in the following Dove Press journal:

Biologics:Targets and Therapy

18 June 2012

Number of times this article has been viewed

\author{
Judith Meza-Junco \\ Michael B Sawyer \\ Department of Oncology, Cross \\ Cancer Institute, Edmonton, \\ Alberta, Canada
}

Correspondence: Judith Meza-Junco Department of Oncology, University of Alberta Cross Cancer Institute, I I 560 University Avenue, Edmonton T6G IZ2, Alberta, Canada $\mathrm{Tel}+\mathrm{I} 7805778125$

Fax +I 7804328888

Email judith.meza-junco@

albertahealthservices.ca

\begin{abstract}
Gastric cancer (GC) is currently the second leading cause of cancer death worldwide; unfortunately, most patients will present with locally advanced or metastatic disease. Despite recent progress in diagnosis, surgery, chemotherapy, and radiotherapy, prognosis remains poor. A better understanding of GC biology and signaling pathways is expected to improve GC therapy, and the integration of targeted therapies has recently become possible and appears to be promising. This article focuses on anti-Her-2 therapy, specifically trastuzumab, as well as other epidermal growth factor receptor antagonists such as cetuximab, panitumub, matuzumab, nimotzumab, gefitinib, and erlotinib. Additionally, drugs that target angiogenesis pathways are also under investigation, particulary bevacizumab, ramucirumab, sorafenib, sunitinib, and cediranib. Other targeted agents in preclinical or early clinical development include mTOR inhibitors, anti c-MET, polo-like kinase 1 inhibitors, anti-insulin-like growth factor, anti-heat shock proteins, and small molecules targeting Hedgehog signaling.
\end{abstract}

Keywords: gastric cancer, targeted therapy, antiangiogenesis drugs, anti-EGFR drugs

\section{Introduction}

Gastric cancer (GC) is very common and is the second leading cause of cancer deaths worldwide. ${ }^{1}$ Approximately $65 \%$ of gastric cancer patients present with locally advanced or metastatic disease, and the majority will have systemic disease at some time during the course of their illness. ${ }^{2}$ For these patients, median overall survival (OS) is about 3 months with best supportive care (BSC) alone. Using combination chemotherapy results in a median OS of 9-14 months. ${ }^{3}$ There is no international consensus regarding the optimal first-line chemotherapy regimen, however a platinum plus fluoropyrimidine doublet or a triplet regimen with the addition of epirubicin or docetaxel are most frequently used. ${ }^{4,5}$

The emergence of new chemotherapy agents and targeted therapies, together with increasing knowledge of biological pathways underlying GC and the ability to predict which patients or tumors will respond to which treatment, may lead to improved GC patient outcomes. ${ }^{6}$ Targeted therapies have emerged as a new hope in cancer management during recent years; several drugs have been studied in GC and these are summarized in Table 1. Most focus on antiangiogenic agents and epidermal growth factor receptor (EGFR) antagonists. Additionally, other molecularly targeted agents are under evaluation in preclinical, phase I, and phase II trials, including matrix metalloproteinases (MMP), c-Met and downstream signaling inhibitors, as well as drugs that target the cell cycle. ${ }^{6}$ 
Table I Targeted therapies for advanced gastric cancer

\begin{tabular}{|c|c|c|}
\hline Targets & Drugs & Trade mark/company \\
\hline \multicolumn{3}{|c|}{ Antiangiogenic therapy } \\
\hline Anti-VEGF & Bevacizumab & Avastin ${ }^{\circledR} /$ Roche \\
\hline Humanized $m A B$ & Ramucirumab & IMC-II2IB/ImClone Systems \\
\hline \multirow[t]{3}{*}{ VEGFR TKI } & Cediranib & Recentin ${ }^{\circledR} /$ AstraZeneca \\
\hline & Sorafenib & Nexavar ${ }^{\circledast / B a y e r}$ \\
\hline & Sunitinib & Sutent ${ }^{\circledR / P f i z e r}$ \\
\hline \multicolumn{3}{|l|}{ Anti EGFR therapy } \\
\hline \multicolumn{3}{|l|}{ EGFR antibody } \\
\hline $\begin{array}{l}\text { Chimeric (mouse/ } \\
\text { human) } m A B\end{array}$ & Cetuximab & Erbitux $^{\circledast / B r i s t o l-M y e r s ~ S q u i b b ~}$ \\
\hline Humanized $m A B$ & Panitumumab & Vectibix ${ }^{\circledast / A m g e n ~}$ \\
\hline Humanized $m A B$ & Matuzumab & EMD 72000/Merk \\
\hline \multirow[t]{2}{*}{ Humanized $m A B$} & Nimotuzumab & TheraCIM ${ }^{\circledR} /$ CIMYM \\
\hline & & Biosciences \\
\hline \multirow[t]{3}{*}{ EGFR TKI } & Gefitinib & Iressa ${ }^{\circledR} /$ AstraZeneca \\
\hline & Erlotinib & Tarceva ${ }^{\circledR} /$ Genentech and \\
\hline & & OSI Pharmaceuticals \\
\hline Her-2 humanized $m A B$ & Traztuzumab & Herceptin ${ }^{\circledast} /$ Roche \\
\hline \multirow[t]{2}{*}{ Her-2 TKI } & Lapatinib & Tykerb ${ }^{\circledR} /$ Tyverb $^{\circledR} /$ \\
\hline & & GlaxoSmithKline plc \\
\hline
\end{tabular}

Abbreviations: VEGF, vascular endothelial growth factor; TKI, tyrosine kinase inhibitor; EGFR, epidermal growth factor receptor; mAbs, monoclonal antibodies.

The goal of this article is to review the development of targeted therapy for advanced GC, with a focus on antiangiogenic, anti-EGFR, and anti-Her-2 drugs.

\section{Antiangiogenesis therapy}

Tumor growth and metastasis has been strongly linked with angiogenesis in most human tumors. Vascular endothelial growth factor (VEGF) is the most potent and specific angiogenic factor identified. The VEGF family includes VEGF-A, -B, -C, -D, and -E, and placenta growth factor (PGF). VGFR-A binds to VEGFR-1 and 2, while VEGF-B and PGF bind to VEGFR-1, and VEGF-C and D bind to VEGFR-2 and 3. Activation of these receptors stimulates a signaling cascade resulting in endothelial cell mitogenesis and migration, induction of proteinases, extracellular matrix remodeling, increased vascular permeability, and maintenance of survival for newly formed blood vessels (Figure 1). ${ }^{7}$ VEGF expression in tumors and VEGF serum levels have been positively correlated with vascular involvement, lymph node and distant metastasis, and poor outcome in advanced GC patients. ${ }^{8}{ }^{8}$ Multiple strategies have been developed to inhibit the VEGF pathway, with most targeting VEGF and its receptor via monoclonal antibodies (mAbs) or tyrosine kinase inhibitors (TKIs).

\section{Anti-VEGF mAbs}

Bevacizumab is a recombinant humanized anti-VEGF-A $\mathrm{mAb}$ that is being evaluated in several tumor types. It is currently approved for colorectal and lung cancer treatment, and phase II studies and a recent phase III trial have studied its efficacy for treatment of advanced GC patients (Table 2).

Shah et al reported response rates (RR) of $67 \%$, a time to progression (TTP) of 8.3 months, and a median OS of 12.3 months in 47 patients treated with bevacizumab combined with cisplatin and irinotecan. ${ }^{10}$ Bevacizumabrelated toxicities were gastric perforation (6\%), myocardial infarction (2\%), and thromboembolic events (25\%).

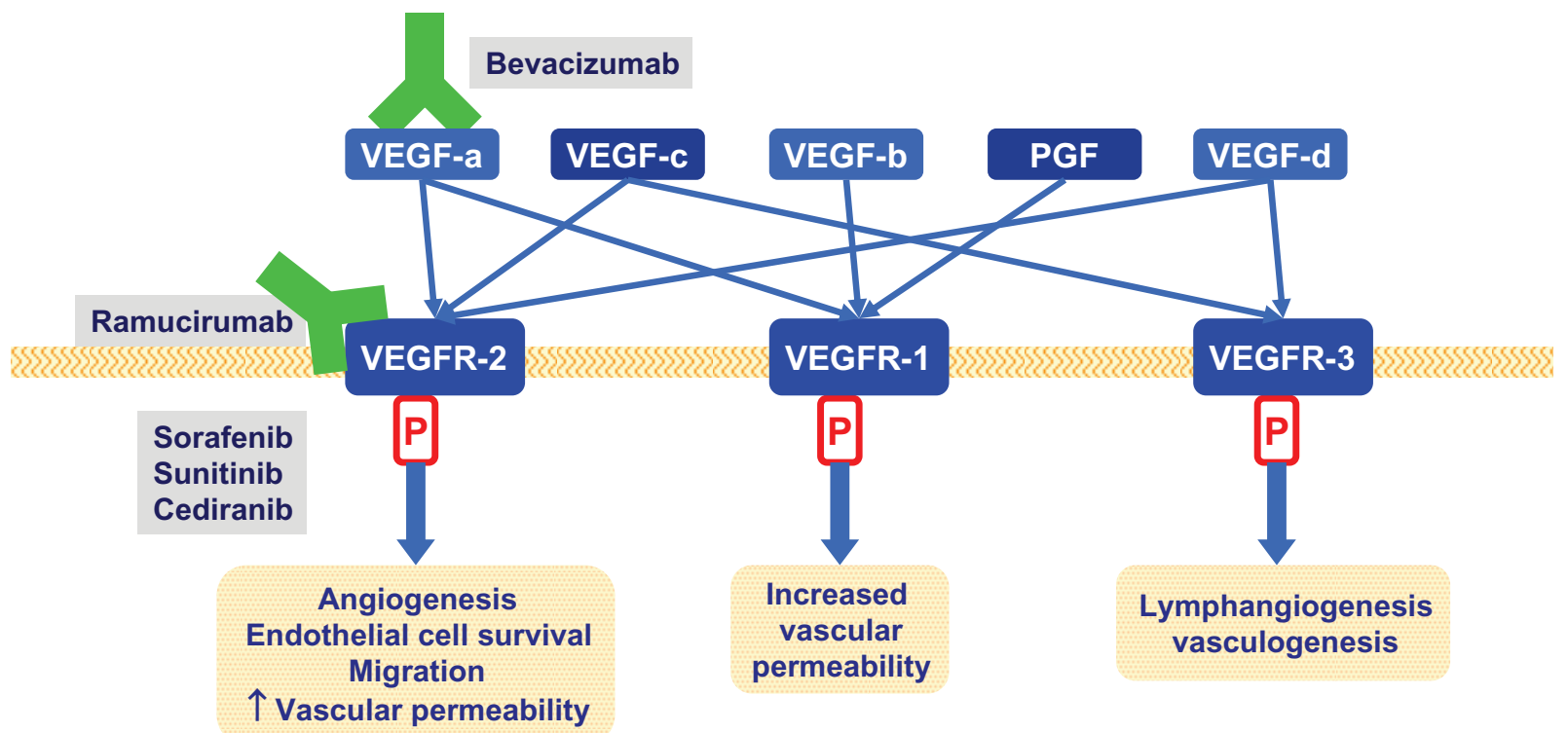

Figure I Relevant anti VEGF pathways therapies in advanced gastric cancer.

Abbreviations: VEGF, vascular endothelial growth factor; VEGFR, vascular endothelial growth factor receptor; PGF, placenta growth factor. 
Table 2 Phase II and III studies of bevacizumab combined with chemotherapy for advanced GC in a first-line setting

\begin{tabular}{|c|c|c|c|c|c|c|}
\hline $\begin{array}{l}\text { Author/type } \\
\text { of study }\end{array}$ & $\mathbf{N}$ & Bevacizumab + chemotherapy & $\% \mathbf{R R}$ & $\begin{array}{l}\text { TTP } \\
\text { months }\end{array}$ & $\begin{array}{l}\text { OS } \\
\text { months }\end{array}$ & $\begin{array}{l}\text { Grade } 3-4 \\
\text { toxicity (\%) }\end{array}$ \\
\hline Shah et $\mathrm{al}^{10}$ & 47 & Cisplatin/irinotecan & 65 & 8.3 & 12.3 & HT: 28, TEE: 25 \\
\hline Phase II & & & & & & GIP: 6, MI: 2 \\
\hline Shah et al" & 44 & Docetaxel/cisplatin/5-FU & 67 & 12 & 16.2 & NTP: 50, Fatigue: 5 \\
\hline Phase II & & & & & & TEE: $39, \stackrel{\perp}{ }$ Other: $<10$ \\
\hline Enzinger et al ${ }^{12,}$ & 26 & Docetaxel/cisplatin/irinotecan & 24 & NA & NA & NTP: 22, Diarrhea: 28 \\
\hline Phase II & & & & & & Nausea: 6, TEE: 9 \\
\hline El-Reyes et $\mathrm{al}^{13}$ & 38 & Oxaliplatin/docetaxel & 59 & 66 & II.I & NTP: 34 \\
\hline Phase II & & & & & & GIP: 8 \\
\hline Cohenuram and Lacy ${ }^{14}$ & 16 & FOLFOX & 63 & 7 & 8.9 & TEE: 0, GIP: 0 \\
\hline \multicolumn{7}{|l|}{ Retrospective } \\
\hline Ohtsu et al ${ }^{15, *}$ & 387 & Bevacizumab + cisplatin/capecitabine & $46^{\ominus}$ & $6.7^{\S}$ & 12. $\left.\right|^{\Pi}$ & TEE: 7, GIP: 2 \\
\hline Phase III & 387 & Placebo + cisplatin/capecitabine & 37.4 & 5.3 & 10.1 & TEE: 7, GIP: 2 \\
\hline
\end{tabular}

Notes: $*$ AVAGAST; ${ }^{\ominus} P=0.0315$; ${ }^{\text {SPFS } P}=0.0037 ;{ }^{n} P=0.1002$; ${ }^{\circ}$ tumor partial responses were $63 \%$ and stable disease $30 \%$; ${ }^{\perp}$ other includes gastrointestinal toxicity, neuropathy, and febrile neutropenia.

Abbreviations: RR, response rate; TTP, time to progression; OS, overall survival; PFS, progression free survival; NA, data is not available; HT, hypertension; TEE, thromboembolic events; MI, myocardial infarction; GIP, gastrointestinal perforation.

The same research group has reported on bevacizumab in combination with docetaxel, cisplatin, and 5-fluorouracil (DCF) in 44 patients. RR were $67 \%$, median progression free survival (PFS) 12 months, and median OS 16.2 months; 2-year OS was 37\%. ${ }^{11}$ Enzinger et al reported similar outcomes with bevcizumab combined with docetaxel, cisplatin, and irinotecan, with partial response (PR) and stable disease (SD) rates of $63 \%$ and $30 \%$, respectively; thromboembolic events were seen in $9 \%$ of patients. ${ }^{12}$

El-Rayes et al treated 38 patients with docetaxel and oxaliplatin plus bevacizumab, achieving a median PFS and OS of 6.6 and 11.1 months, respectively. ${ }^{13}$ Complete responses $(\mathrm{CR})$ were seen in $5 \%$, PR in $37 \%$, and $\mathrm{SD}$ in $37 \%$ of patients. Gastrointestinal perforation occurred in $8 \%$ of patients.

Cohenuram and Lacy found less toxicity and similar RR when bevacizumab was combined with 5 -fluorouracil (5-FU), leucovorin, and oxaliplatin (FOLFOX) in 16 patients. ${ }^{14} \mathrm{PR}$ and SD were seen in $63 \%$ and $37 \%$ of patients, respectively, and TTP and OS were 7 and 8.9 months, respectively. In contrast to the studies discussed above, no bevacizumab-related toxicity was seen.

Based on these promising results, a phase III, doubleblind, randomized, and multicenter study of bevacizumab versus placebo in combination with capecitabine and cisplatin, as first-line therapy in advanced GC patients (AVAGAST), ${ }^{15}$ was recently published. This failed to show a difference in OS, with median OS 12.1 versus 10.1 months for the bevacizumab arm versus the placebo arm, respectively $(\mathrm{HR}=0.87 ; 95 \% \mathrm{CI}=0.73-1.03 ; P=0.1002)$. However, PFS was 6.7 versus 5.3 months ( $\mathrm{HR}=0.80 ; 95 \%$ $\mathrm{CI}=0.68-0.93 ; P=0.0037)$ and $\mathrm{RR}$ was 46.0 versus $37.4 \%$
$(P=0.0315)$, both favoring the bevacizumab treatment arm. Most common grade 3-5 adverse events including neutropenia, anemia, and decreased appetite were similar in the two groups. Incidence of grade 3-5 (events potentially related to bevacizumab) was 20 versus $15 \%$ in the placebo group. Thromboembolic events occurred in $7 \%$ of patients and gastrointestinal perforation occurred in $2 \%$ of patients in both arms. A preliminary report of AVAGAST biomarker analysis performed in 763 tumor and 712 plasma samples showed that a low tumor neutropilin (a co-receptor for VEGF-A) expression was associated with shorter OS in placebo-treated patients. ${ }^{16}$

\section{Anti-VEGFR mAbs}

Ramucirumab is a fully human, IgG1 mAbs that inhibits VEGFR-2. It is currently under investigation in phase III studies as second-line chemotherapy for advanced GC. Phase I clinical trials demonstrated its safety and efficacy in patients with advanced cancer refractory to standard chemotherapy. ${ }^{17}$ The phase III trial RAINBOW is now comparing paclitaxel plus ramucirumab or placebo, while another phase III trial is recruiting patients to receive ramucirumab or BSC.

\section{VEGFTKI}

Sorafenib is an oral multitargeted TKI that inhibits VEGFR-1, VEGFR-2, VEGFR-3, platelet derived growth factor (PDGFR), B-Raf, Raf-1, and c-Kit. Sorafenib combined with capecitabine and cisplatin was tested for advanced GC in a phase I study, achieving an encouraging RR of $62.5 \%$, PFS of 10 months, and OS of 14.7 months..$^{18} \mathrm{~A}$ subsequent phase II study of sorafenib with 3-weekly docetaxel and cisplatin resulted in OS of 13.6 months but a PFS of 5.8 months, which 
is less than that reported in a phase III study of chemotherapy alone. This could suggest that the longer OS duration may reflect the use of second-line chemotherapy. ${ }^{19}$

Sunitinib is also a multitargeted TKI (targeting RET, VEGFR-1, VEGFR-2, VEGFR-3, PDGFR $\alpha$, PDGFR $\beta$, Flt 3 , c-KIT, and colony-stimulating factor receptor 1 ), which has been tested as monotherapy for second-line treatment of advanced GC showing modest activity. In a study involving 78 patients, Bang et al reported minimal radiological RR (2.6\%), and PFS and OS of 2.3 and 6.8 months, respectively. ${ }^{20}$ Similar outcomes were found by Moehler et al in 51 patients, with RR of $3.9 \%$, PFS and OS of 1.28 and 5.81 months, respectively, and estimated 1-year OS of $23.7 \%{ }^{21}$ In subgroup analyses, tumor VEGF-C expression compared with no expression was associated with significantly shorter median PFS (1.23 versus 2.86 months, $P=0.0119)$ but there was no difference in $\mathrm{RR}(P=0.142)$.

Cediranib (VEGFR TKI) in combination with cisplatin and S-1 or capecitabine was tested in 14 patients as a firstline treatment. The most common adverse events were decreased appetite, fatigue, and nausea (92.9\%). Preliminary efficacy evaluation showed one confirmed and three unconfirmed PR.22

Additional studies with sorafenib, sunitinib, and cediranib are needed in advanced GC. Other VEGFR TKIs such as apatinib, axitinib, vatalinib, semaxinib, vandetanib, and pazopanib, as well as aflibercept (anti VGFR-A and placenta growth factor), MNRP1685A (anti-neuropilin-1 antibody), and PX-478 (oral inhibitor of hypoxia inducible factor- $1 \alpha$ ) are under investigation in phase I and II trials in patients with advanced solid tumors, but none of these agents have yet been evaluated in $\mathrm{GC}$ cancer.

\section{Biomarkers and resistance mechanism of antiangiogenesis therapy}

Despite extensive preclinical and clinical research there are currently no validated biomarkers to select patients for antiangiogenic therapy, although some candidate surrogate markers of bevacizumab response have been described. Tumor VEGF expression was identified as a poor prognosis marker in GC patients. There was a significant correlation between VEGF expression and tumor lymphatic and vascular invasion, lymph node and liver metastases, and OS. ${ }^{23}$ VEGF-C, VEGF-D, and VEGFR-3 expression have also been found to be independent prognostic markers for OS in resected GC patients. ${ }^{23,25}$ Bernaards et al reported a preliminary analysis of phase III studies including three tumor types, and found that high levels of circulating VEGF correlated with shorter
PFS and OS times independently of bevacizumab treatment. ${ }^{26}$ Polymorphisms in genes encoding proteinase activated receptor 1 (PAR-1-506) and EGF (EGF+61 A > G (A/A)), which are receptors involved in VEGF regulation, were correlated with a higher risk for disease recurrence in resected esophageal cancer patients. Circulating endothelial progenitor cells (CEPs) and circulating endothelial cells (CECs) may regulate the angiogenic switch, promoting angiogenesis and micrometastases progression, and while CEPs have been described in patients with advanced GC undergoing chemotherapy their role as predictive biomarkers needs a prospective evaluation. ${ }^{27}$ Hypertension has been correlated with longer PFS and OS in a retrospective analysis of a phase III study of interferon with or without bevacizumab for renal cell carcinoma patients. ${ }^{28}$ Imaging methods such as CT scan, MRI, and PET scan may assist in the early treatment response of patients treated with antiangiogenic therapy.

Preclinical and clinical studies demonstrate resistance to antiangiogetic therapy affecting patient OS. Moreover, it is evident that the angiogenesis inhibitors that are currently available are not providing long-term efficacy, and drug resistance commonly develops after a few months of therapy. The resistance mechanisms of antiangiogenesis drugs are not completely known, and most investigations have been done in pre-clinical studies with animals so do not necessarily mirror what has been observed in the clinic. Reports suggest that hypoxia generated by angiogenesis inhibition or the blockage of new blood vessels triggers signaling molecules that make tumors more aggressive and metastatic. The theory of rebound means that by cutting off oxygen to cancer cells, angiogenesis inhibition eventually forces those cells to migrate to other, nonhypoxic locations. Hypoxia induces this effect by stimulating hepatocyte growth factor (HGF, which normally controls cell growth, migration, and shape) and c-Met (which is HGF's cell surface receptor) - this means that when one angiogenic factor is knocked out another takes its place. Preclinical models also suggest that targeting VEGF and blocking c-Met is an option to improve outcomes with antiangiogenic treatment, as this may inhibit agiogenesis while avoiding an increase in hypoxia, so preventing a change in tumor biology that makes it more aggressive. ${ }^{29-31}$ Thus, better efficacy may be achieved through the use of combinations of drugs targeting different angiogenic growth factors, or drugs such as sorafenib and sunitinib that target multiple angiogenesis pathways. Another strategy may be obtaining tumor biopsies to analyze growth factors that are upregulated in a particular patient in order to administer a more specific or tailored antiangiogenesis treatment. ${ }^{32}$ Resistance mechanisms 
of antiangiogenesis therapy are an extensive and complex topic that have been discussed in recent reviews. ${ }^{29-31}$

\section{Conclusions}

Angiogenesis inhibitors are an important class of anti-cancer agents, but the response to current treatments is transient, and more research is required to address why drug resistance occurs. Although extensive research has been done on angiogenesis mechanisms in cancer patients, many aspects remain unknown, and further clinical research is needed to identify clinical activity, predictors of response, toxicity, and optimal therapy duration. Antiangiogenic agents have some activity in GC cancer patients, but no trial has proved beneficial in terms of OS.

\section{Anti-EGFR therapy}

EGFR belongs to the ERBB family of four related cell-surface receptors: ERBB1, 2 (Her-2/neu), 3 and 4. Ligand binding to the extracellular domain (ECD) leads to EGFR activation, after phosphorylation of the intracellular tyrosine kinase (TK) initiates downstream signaling cascades including the central Ras/Raf/mitogen-activated protein kinase (MAPK, which is mainly involved in cell proliferation) and the Akt/mTOR pathway, which is mainly involved in cell survival and motility invasion. ${ }^{33} \mathrm{mAb}$ that target the extracellular domain of EGFR (panitumumab, cetuximab, matuzumab, and nimotuzumab) are currently under evaluation in advanced GC. Gefitinib and erlotinib are small molecule inhibitors directed against the intracellular TK domain of EGFR, and have undergone phase I and II evaluation. Lapatinib, another TKI that targets Her-2 and EGFR, is currently undergoing phase III evaluation. Anti-Her-2 mAb (trastuzumab) is a standard treatment for advanced GC with Her-2 overexpression or gene amplification. Everolimus inhibits the intracellular downstream signaling protein mTOR, and is in phase III evaluation (Figure 2). ${ }^{33}$

EGFR expression in GC has been reported to be between 18 and $81 \%$, variation which is determined almost exclusively by gene amplification. Overexpression seems to be associated with increased invasion, a more poorly differentiated histology, and shorter survival, ${ }^{34}$ but evidence regarding potential prognostic roles of EGFR is limited and controversial.

\section{Anti-EGFR mAbs}

Cetuximab binds to the ECD of EGFR on normal and tumor cells, and competitively inhibits the binding of epidermal
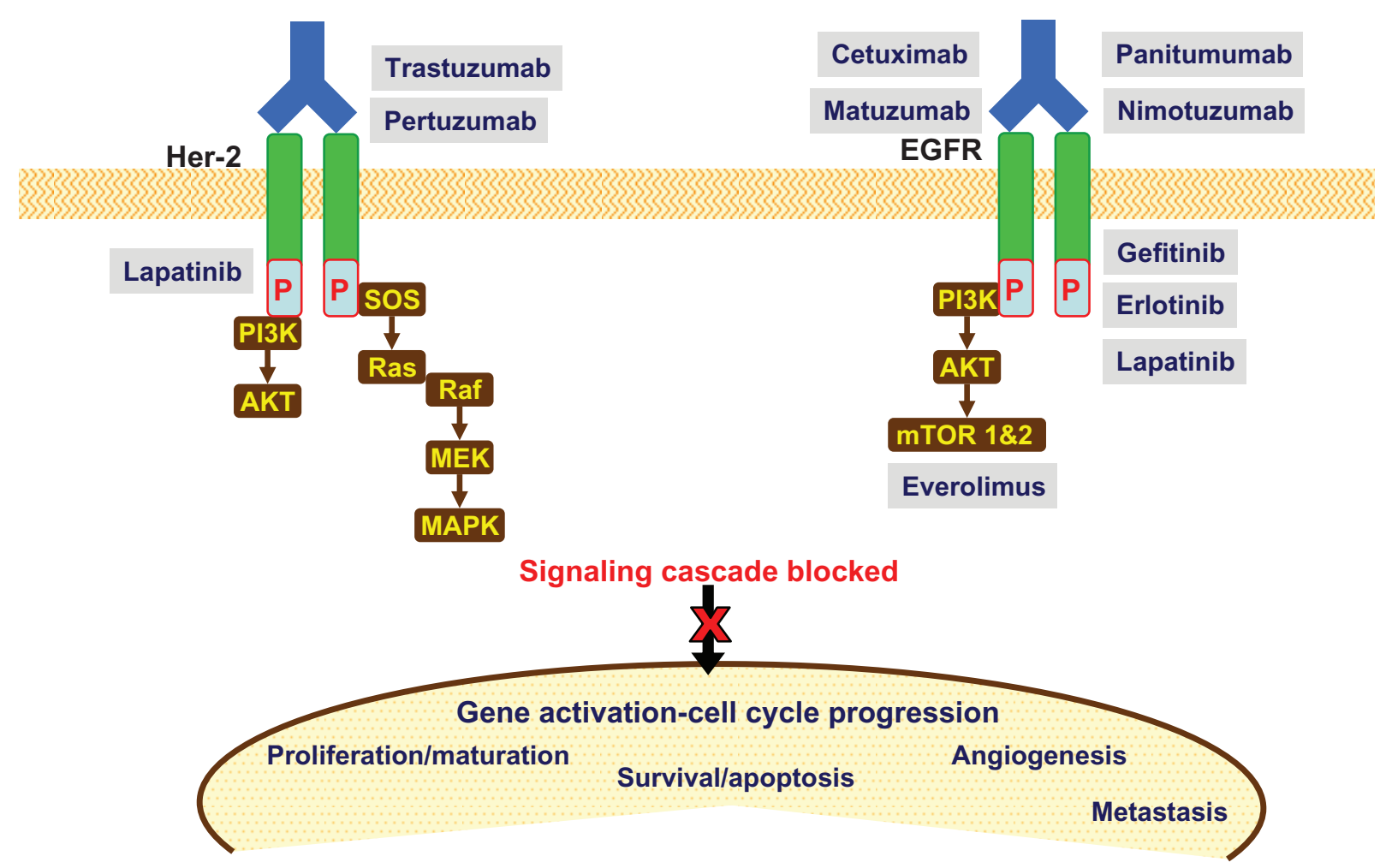

Figure 2 Molecular targets and relevant drugs (anti-EGFR/Her-2) in advanced gastric cancer.

Abbreviations: AKT, protein kinase B; MAPK, mitogen-activated protein kinase; MEK, MAP kinase kinase; mTOR I\&2, mammalian target of rapamycin I\&2; PI3K, phosphatidylinositol 3 kinase; Raf, GTPase Raf; Ras, GTPase Ras; SOS, son of sevenless homolog. 
growth factor (eEGF) and other ligands such as transforming growth factor-alpha (TGF) and amphiregulin. It has also been shown to mediate antibody-dependent cell cytotoxicity (ADCC). Cetuximab has been studied in several phase II trials including in patients with advanced GC in different lines of treatment. ${ }^{34}$

Several first-line treatment phase II trials evaluated the activity and safety of cetuximab combined with different chemotherapy regimens such as FOLFIRI, docetaxel/ cisplatin, FOLFOX, FUFOX, XELOX, FUFIRI, continuous infusion high dose 5-FU/leucovorin/cisplatin, capecitabine/ cisplatin, and oxaliplatin/irinotecan. RR varied from 41 to $69 \%$, TTP from 5 to 8.5 months, and OS from 9 to 16.6 months. Skin rash, diarrhea, nausea/vomiting, and infusion reactions were the most serious cetuximab-related side effects reported. The relationship between RR and EGFR is not well established, and contradictory results have been reported. ${ }^{34,35}$

Phase III randomized trials are underway to assess options for first-line of treatment in advanced GC (Table 3). The CALGB80403/ECOG 1206 study is comparing cetuximab combined with three different chemotherapy regimens (ECF versus irinotecan plus cisplatin [IC] versus FOLFOX). Preliminary reports showed RR of 57.8, 45.6, and $53.6 \%$, respectively, and OS of $11.5,8.9$, and 12.4 months, respectively. Cetuximab combined with FOLFOX had one of the most favorable safety profiles. ${ }^{36}$ The EXPAND trial is currently studying capecitabine and cisplatin with/without cetuximab.
In second-line setting, cetuximab monotherapy has been shown to have minimal RR and an OS of 3.1 months $^{37}$ to 4 months. ${ }^{38}$ When it was combined with docetaxel in docetaxel-refractory patients, RR was $6 \%$ and PFS was 2.1 months. ${ }^{39}$ The combination of cetuximab and irinotecan in heavily pre-treated patients achieved a tumor control rate of $62 \%$ (eight patients), and TTP and OS were 78 and 101 days, respectively. ${ }^{40}$

Matuzumab is being tested in a phase II randomized trial (MATRIX) comparing ECX with/without matuzumab. Preliminary results show an RR of 58 versus $31 \%$, TTP of 7.1 versus 4.8 months, and OS of 12.2 versus 9.4 months, all favoring the group receiving matuzumab. ${ }^{41}$

REAL-III, an ongoing phase III trial, is being carried out to determine whether adding panitumumab to standard chemotherapy (EOX) improves the survival of patients with advanced GC.

A randomized phase II study of irinotecan with/ without nimotuzumab in patients previously treated showed a median PFS time of 73 days with the addition of nimotzumab as opposed to 85 days with irinotecan alone. Preliminary results of this trial show that $44 \%$ of 48 samples analyzed had positive staining for EGFR, with a trend towards a potential benefit of nimotuzumab in this subgroup of patients. ${ }^{42}$

\section{EGFR TKI}

Erlotinib sensitivity is dependent on tumor location. SWOG showed no tumor responses in patients with GC, while

Table 3 Anti EGFR therapy for advanced gastric cancer

\begin{tabular}{|c|c|c|c|c|c|c|}
\hline Study/phase & Treatment & $\mathbf{N}$ & $\% \mathbf{R R}$ & $\begin{array}{l}\text { TTP } \\
\text { months }\end{array}$ & $\begin{array}{l}\text { OS } \\
\text { months }\end{array}$ & $\begin{array}{l}\text { Grade } 3-4 \\
\text { toxicity (\%) }\end{array}$ \\
\hline CALGB 80403/ & $E C F+$ cetuximab & 67 & 57.8 & 5.9 & 11.5 & Hem: 5I, nonHem: 60 \\
\hline \multirow[t]{2}{*}{ ECOG $1206^{36}$} & $I C+$ cetuximab & 7I & 45.6 & 5 & 8.9 & Hem: 56, nonHem: 70 \\
\hline & FOLFOX + cetuximab & 72 & 53.6 & 6.7 & 12.4 & Hem: 45, nonHem: 64 \\
\hline MATRIX $^{41}$ & $\mathrm{ECX}$ & 36 & 58 & 7.1 & 12.2 & NTP: 33, SD: 33 \\
\hline Phase II & $\mathrm{ECX}+$ matuzumab & 35 & 31 & 4.8 & 9.4 & NTP: 37, SD: 66 \\
\hline \multirow[t]{2}{*}{ REAL III } & EOX & NA & NA & NA & NA & NA \\
\hline & EOX + chemotherapy & & & & & \\
\hline Phase II ${ }^{42}$ & Irinotecan & 40 & NA & 73 days* & 293 days & NA \\
\hline second-line & Irinotecan + nimotuzumab & 42 & & 85 days* & 227 days & \\
\hline ToGA ${ }^{51}$ & Capecitabine/5-FU + cisplatin + trastuzumab & 298 & NA & $6.7^{*}$ & 13.5 & Overall: $68, \mathrm{CE}: 6$ \\
\hline Phase II & Capecitabine/5-FU + cisplatin & 296 & & $5.5^{*}$ & II.I & Overall: $68, \mathrm{CE}: 6$ \\
\hline TYTAN $^{56, \theta}$ & Paclitaxel + lapatinib & NA & NA & NA & NA & NA \\
\hline Phase III & Paclitaxel & & & & & \\
\hline \multirow[t]{2}{*}{$\mathrm{LOGiC}^{57}$} & Capecitabine + oxaliplatin + lapatinib & NA & NA & NA & NA & NA \\
\hline & Capecitabine + oxaliplatin + placebo & & & & & \\
\hline
\end{tabular}

Notes: *Progression free survival; ${ }^{\ominus}$ second line of treatment.

Abbreviations: Hem, hematological toxicity; NTP, neutropenia; OS, overall survival; SD, skin disorders; CE, cardiac events; RR, reported response rates; TTP, time to progression. 
patients with gastroesophageal junction cancer had an RR of $9 \%$. OS was 3.5 and 6.7 months and TTP was 1.6 and 3 months, respectively, for gastric and gastroesophageal locations. ${ }^{43}$

Gefitinib has been evaluated in a phase II study aimed to assess biologic activity of EGFR TKI in 70 previously treated advanced GC patients, and gefitinib reached tumor concentrations sufficient to inhibit EGFR activation, although this did not translate into clinical benefit. ${ }^{44}$ In patients with locally advanced esophageal and gastroesophageal junction cancer, gefitinib combined with cisplatin+5-FU and radiotherapy as a neoadjuvant treatment did not increase pathological CR rates, but 3-year OS increased compared with historical controls (42\% versus $28 \%)^{45}$

The lack of erlotinib and gefitinib activity in GC in these trials may be related to the different etiology seen in different tumor locations (gastroesophageal junction adenocarcinomas are associated with Barrett's esophagus, and GC with Helicobacter pylori infection). The different molecular pathways targeted by EGFR inhibitors may be differentially expressed in proximal versus distal adenocarcinomas. Moreover, gefitinib and erlotinib may have a different repertoire of receptor inactivation.

\section{Anti-Her-2 therapy}

Her-2/neu (ERBB2) is a member of the ERBB TK receptor family. Trastuzumab is an $\mathrm{mAb}$ and lapatinib is a TKI; both target Her-2 and are currently under clinical development in GC. Her-2 overexpression (evaluated by immunohistochemistry (IHC) and fluorescent in situ hybridization (FISH) analysis) has been reported in $6-45 \%$ of GC. ${ }^{46,47}$ The largest data set of 3883 advanced GC samples found Her-2 positivity in $22.9 \%$ of samples. Her- 2 positivity was higher in gastroesophageal junction cancers than GC (33.2 versus $20.9 \%, P<0.001$ ), and higher in intestinal than in diffuse/mixed cancer (32.2 versus $6.1 \% / 20.4 \%$, $P<0.001)^{48}$

The prognostic role of Her-2 in GC remains uncertain, since some authors have not found any association between Her-2 expression and OS, while many others have shown significant correlations between elevated Her-2 expression and both more aggressive disease and worse outcomes. ${ }^{46}$

\section{Anti-Her-2 mAbs}

Preclinical studies with GC cell lines that overexpress Her-2 showed growth-inhibitory effects of trastuzumab, but when trastuzumab was combined with doxorubicin, cisplatin, or paclitaxel there was increased cytotoxicity. ${ }^{49}$ The preliminary results of a small phase II study including 21 advanced GC patients with Her-2 overexpression demonstrated RR of $35 \%, 17 \%$ of the group had stable disease (SD), and it was well tolerated..$^{50}$

The ToGA study is the first phase III, randomized and controlled trial to evaluate trastuzumab efficacy and safety in Her-2-positive advanced GC. In this study, 594 patients received either trastuzumab in combination with $5 \mathrm{FU} /$ capecitabine and cisplatin, or chemotherapy alone. OS was 13.5 versus 11.1 months $(P=0.0048)$ respectively, with a $26 \%$ reduction in the risk of death. PFS was 6.7 versus 5.5 months, respectively $(P=0.0002)$, and disease control was 47.3 versus $34.5 \%$, respectively $(P=0.0017)$. Safety profiles were similar in both groups. Interestingly, patients with high IHC positivity for Her-2 had a trend for better OS, while patients with Her-2 IHC2+/FISH+ or IHC3+ had a longer OS (16 months) with trastuzumab compared to chemotherapy alone (11.8 months) ${ }^{51}$ Trastuzumab seems to be a reasonable treatment option for Her-2-positive advanced GC patients, although only approximately $20 \%$ of patients would be potential candidates.

\section{Her-2 TKI}

Lapatinib is a dual inhibitor of EGFR and Her-2. The SWOG-S0413 trial evaluated first-line lapatinib monotherapy in 47 patients, achieving modest activity. Only $7 \%$ of patients had a PR, and $20 \%$ had SD. TTP was 2 months and OS 5 months. ${ }^{52}$ In pretreated patients, no objective response was seen in 21 patients. ${ }^{53}$ When lapatinib is combined with capecitabine as a first-line treatment, the regimen had an $\mathrm{RR}$ of $24 \%$ in 58 advanced GC patients. ${ }^{54} \mathrm{~A}$ randomized, placebo-controlled phase II study (EORTC-40071) to evaluate lapatinib in combination with ECF or ECX is ongoing, and this will also prospectively explore the roles of Her-2 and EGFR status. ${ }^{55}$

Lapatinib is currently being tested in TYTAN, an openlabel, randomized phase III study comparing paclitaxel with and without lapatinib in advanced GC patients expressing Her-2 as second-line therapy. ${ }^{56}$ The LOGiC trial will compare capecitabine and oxaliplatin with/without lapatinib in a first-line setting. ${ }^{57}$

\section{Biomarkers and resistance mechanism of anti-EGFR therapy}

Most of the information available regarding biomarkers and resistance mechanisms of anti-EGFR drugs comes 
from colorectal cancer. The presence of somatic KRAS mutations has been clearly identified as a predictive marker of resistance to anti-EGFR in colorectal cancer, and the use of these drugs is restricted to patients with no detectable KRAS mutations. Several studies have indicated that amplification/overexpression of EGFR and inactivation of the anti-oncogene TP53 are associated with sensitivity to anti-EGFR mAbs, whereas mutations of $B R A F$ and PIK $3 C A$ and loss of PTEN expression are associated with resistance. Besides these somatic variations, germline polymorphisms (affecting genes involved in the EGFR pathway or within the immunoglobulin receptors) may also modulate response to anti-EGFR mAbs. These markers are not completely validated at this time, and only KRAS genotyping is mandatory in routine clinical practice of anti-EGFR mAbs in colon cancer..$^{58-60}$

The role of EGFR inhibition in advanced GC is not completely clear, but may be established by ongoing clinical trials such as the REAL III trial (EOX chemotherapy with/ without panitumub).

Predicting metabolic responders by PET scan may have a role in GC patients receiving cetuximab, ${ }^{61}$ but large and prospective trials have yet to be performed. Mutations in the KRAS gene have been shown in multiple phase III trials to predict lack of efficacy in patients with metastatic colorectal cancer, but these results may not be extrapolated to other tumors. Prevalence of KRAS mutations in GC cancer is reported to be approximately $3-30 \%,{ }^{62}$ and the REAL III study will be prospectively studying its incidence and effect on panitumumab efficacy. The expression of ligands (epiregulin and amphiregulin) has been described in GC cell lines, ${ }^{63}$ and their role as predictors of response in GC patients has not been evaluated yet.

\section{Conclusions}

EGFR targeting is an area of current clinical research in GC. Promising data regarding the addition of trastuzumab to chemotherapy led to a new standard of care for advanced GC patients who were Her-2 positive. The best biomarkers to predict efficacy of Her-2-targeted therapy is Her-2 protein overexpression and Her-2 gene amplification, but a biomarker to predict trastuzumab resistance is urgently required.

\section{Other targeted therapies}

Other targeted agents are in clinical development:

- Everolimus (mTOR inhibitor) as monotherapy was well tolerated with promising activity in previously treated advanced GC patients $(\mathrm{SD}=56 \%, \mathrm{PFS}=2.7$ months, OS $=10.1$ months).${ }^{64}$ Based on these results, a global randomized phase III study (GRANITE-1) was performed with 656 patients, and showed no significant improvement in OS when patients received everolimus compared with placebo (5.39 versus 4.34 months, respectively $(P=0.1244)$, while PFS was 1.68 versus 1.41 months respectively $(P<0.0001) .{ }^{65}$

- c-MET's receptors and its ligand, HGF, regulate multiple cellular processes that stimulate cell proliferation, invasion, and angiogenesis. Recent research has showed their overexpression in solid tumors including GC. ${ }^{66}$

- Polo-like kinase 1 (Plk1) inhibitors are a new class of drugs that target Plk1, which is involved in different stages of mitosis (centrosome maturation, spindle formation, chromosome separation, and cytokinesis). Because PIk1 is mainly expressed in proliferating tissues and is overexpressed in cancers, its inhibition is potentially less toxic, and several Plk1 inhibitors are being evaluated as cancer treatment drugs. ${ }^{67}$

- Insulin-like growth factor (IGF)-I receptor signaling is required for carcinogenicity and proliferation of gastrointestinal cancers. The effects of the new mAb figitumumab (CP-751,871) are being evaluated. ${ }^{68}$

- Heat shock proteins (HSP) are molecular chaperones for several cellular proteins and have cytoprotective roles. Hsp90, for example, interacts with the proteins that mediate cell signaling involved in proliferation, cell cycle control, angiogenesis, and apoptosis. Several initial clinical studies have shown promising anticancer activity of Hsp90 inhibitors. ${ }^{69}$

- Hedgehog (Hh) signaling is over-activated in several solid tumors, which plays a central role in cell growth, stroma recruitment, and tumor progression. In the Hh signaling pathway, the Smoothened (SMO) receptor comprises a primary drug target, with experimental small molecule SMO antagonists currently being evaluated in clinical trials. $^{70}$

\section{Conclusion}

$\mathrm{GC}$ is an aggressive disease with a high mortality rate, and the majority of GC patients in Western clinical practice have advanced disease. Despite recent progress in diagnosis, surgical techniques, chemotherapy, and radiotherapy, prognosis remains poor. Improvements in GC therapy are expected as our understanding of GC biology and signaling pathways improves, and the integration of targeted therapies is now possible and early results are promising. Better selection of 
the therapy for individual patients may also significantly improve treatment and patient survival.

\section{Disclosure}

The authors report no conflicts of interested in this work.

\section{References}

1. Jemal A, Bray F, Center MM, Ferlay J, Ward E, Forman D. Global cancer statistics. CA Cancer J Clin. 2011;61(2):69-90.

2. Sugano K. Gastric cancer: pathogenesis, screening and treatment. Gastrointest Endosc Clin N Am. 2008;18(3):513-522.

3. Wagner AD, Grothe W, Haerting J, Kleber G, Grothey A, Fleig WE. Chemotherapy in advanced gastric cancer: a systematic review and meta-analysis based on aggregate data. J Clin Oncol. 2006;24(18): 2903-2909.

4. Cunningham D, Starling N, Rao S, et al. Capecitabine and oxaliplatin for advanced esophagogastric cancer. $N$ Engl J Med. 2008;358(1): $36-46$.

5. Van Cutsem E, Moiseyenko VM, Tjulandin S, et al. Phase III study of docetaxel and cisplatin plus fluorouracil compared with cisplatin and fluorouracil as first-line therapy for advanced gastric cancer: a report of the V325 Study Group. J Clin Oncol. 2006;24(31):4991-4997.

6. Power DG, Kelsen DP, Shah MA. Advanced gastric cancer - slow but steady progress. Cancer Treat Rev. 2010;36(5):384-392.

7. Okines AF, Reynolds AR, Cunningham D. Targeting angiogenesis in esophagogastric adenocarcinoma. Oncologist. 2011;16(6):844-858

8. Maeda K, Chung YS, Ogawa Y, et al. Prognostic value of vascular endothelial growth factor expression in gastric carcinoma. Cancer. 1996;77(5):858-863.

9. Yoshikawa T, Tsuburaya A, Kobayashi O, et al. Plasma concentration of VEGF and bFGF in patients with gastric carcinoma. Cancer Lett. 2000;153(1-2):7-12.

10. Shah MA, Ramanathan RK, Ilson DH, et al. Multicenter phase II study of irinotecan, cisplatin, and bevacizumab in patients with metastatic gastric or gastroesophageal junction adenocarcinoma. J Clin Oncol. 2006;24(33):5201-5206.

11. Shah MA, Jhawer M, Ilson DH, et al. Phase II study of modified docetaxel, cisplatin, and fluorouracil with bevacizumab in patients with metastatic gastroesophageal adenocarcinoma. J Clin Oncol. 2011;29(7):868-874.

12. Enzinger P, Ryan D, Regan E, et al. Phase II trial of docetaxel, cisplatin, irinotecan, and bevacizumab in metastatic esophagogastric cancer. J Clin Oncol. 2008;26 Suppl 15:S4552.

13. El-Rayes BF, Zalupski M, Bekai-Saab T, et al. A phase II study of bevacizumab, oxaliplatin, and docetaxel in locally advanced and metastatic gastric and gastroesophageal junction cancers. Ann Oncol. 2010;21(10):1999-2004.

14. Cohenuram MK, Lacy J. FOLFOX6 and bevacizumab (FOLFOX6/B) for metastatic esophageal, gastroesophageal (GE) and gastric cancer (G) adenocarcinoma: a single institution's initial clinical experience. Proceedings of GI ASCO; 2008:74.

15. Ohtsu A, Shah MA, Van Cutsem E, et al. Bevacizumab in combination with chemotherapy as first-line therapy in advanced gastric cancer: a randomized, double-blind, placebo-controlled phase III study. J Clin Oncol. 20 2011;29(30):3968-3976.

16. Shah M, Kang Y, Ohtsu A, et al. Tumor and blood plasma biomarker analyses in the AVAGAST phase III randomized study of first-line bevacizumab + capecitabine/cisplatin in patients with advanced gastric cancer. Ann Oncol. 2010;21 Suppl 8:S176.

17. Spratlin JL, Cohen RB, Eadens M, et al. Phase I pharmacologic and biologic study of ramucirumab (IMC-1121B), a fully human immunoglobulin G1 monoclonal antibody targeting the vascular endothelial growth factor receptor-2. J Clin Oncol. 2010;28(5): 780-787.
18. Kim C, Lee JL, Choi YH, et al. Phase I dose-finding study of sorafenib in combination with capecitabine and cisplatin as a first-line treatment in patients with advanced gastric cancer. Invest New Drugs. 2012;30(1):306-315

19. Sun W, Powell M, O'Dwyer PJ, et al. Phase II study of sorafenib in combination with docetaxel and cisplatin in the treatment of metastatic or advanced gastric and gastroesophageal junction adenocarcinoma: ECOG 5203. J Clin Oncol. 2010;28(18): 2947-2951.

20. Bang YJ, Kang YK, Kang WK, et al. Phase II study of sunitinib as second-line treatment for advanced gastric cancer. Invest New Drugs. 2011;29(6)L1449- L1458.

21. Moehler M, Mueller A, Hartmann JT, et al. An open-label, multicentre biomarker-oriented AIO phase II trial of sunitinib for patients with chemo-refractory advanced gastric cancer. Eur J Cancer. 2011; 47(10):1511-1520.

22. Satoh T, Yamada Y, Muro K, et al. Phase I study of cediranib in combination with cisplatin plus fluoropyrimidine (S-1 or capecitabine) in Japanese patients with previously untreated advanced gastric cancer. Cancer Chemother Pharmacol. 2012;69(2):439-446.

23. Maeda K, Chung YS, Takatsuka S, et al. Tumour angiogenesis and tumour cell proliferation as prognostic indicators in gastric carcinoma. Br J Cancer. 1995;72(2):319 -323.

24. Jüttner $\mathrm{S}$, Wissmann $\mathrm{C}$, Jöns $\mathrm{T}$, et al. Vascular endothelial growth factor-D and its receptor VEGFR-3: two novel independent prognostic markers in gastric adenocarcinoma. J Clin Oncol. 2006;24(2):228-240.

25. Ding S, LiC, Lin S, et al. Distinct roles of VEGF-A and VEGF-C in tumour metastasis of gastric carcinoma. Oncol Rep. 2007;17(2):369-375.

26. Lurje G, Leers JM, Pohl A, et al. Genetic variations in angiogenesis pathway genes predict tumor recurrence in localized adenocarcinoma of the esophagus. Ann Surg. 2010;251(5):857- 864.

27. Ahn JB, Rha SY, Shin SJ, et al. Circulating endothelial progenitor cells (EPC) for tumor vasculogenesis in gastric cancer patients. Cancer Lett. 2010;288(1):124-132.

28. Rini BI, Halabi S, Rosenberg JE, et al. Phase III trial of bevacizumab plus interferon alfa versus interferon alfa monotherapy in patients with metastatic renal cell carcinoma: final results of CALGB 90206. J Clin Oncol. 2010;28(13):2137-2143.

29. Shojaei F, Ferrara N. Role of the microenvironment in tumor growth and in refractoriness/resistance to anti-angiogenic therapies. Drug Resist Updat. 2008;11(6):219-230.

30. Azam F, Mehta S, Harris AL. Mechanisms of resistance to antiangiogenesis therapy. Eur J Cancer. 2010;46(8):1323-1332.

31. Eikesdal HP, Kalluri R. Drug resistance associated with antiangiogenesis therapy. Semin Cancer Biol. 2009;19(5):310-317.

32. Ma J, Waxman DJ. Combination of antiangiogenesis with chemotherapy for more effective cancer treatment. Mol Cancer Ther. 2008;7(12): 3670-3684.

33. Mendelsohn J. Targeting the epidermal growth factor receptor for cancer therapy. J Clin Oncol. 2002;20(Suppl 18):S1-S13.

34. Okines A, Cunningham D, Chau I. Targeting the human EGFR family in esophagogastric cancer. Nat Rev Clin Oncol. 2011;8(8): 492-503.

35. Cappetta A, Lonardi S, Pastorelli D, Bergamo F, Lombardi G, Zagonel V. Advanced gastric cancer (GC) and cancer of the gastrooesophageal junction (GEJ): focus on targeted therapies. Crit Rev Oncol Hematol. 2012;81(1):38-48.

36. Enzinger PC, Burtness B, Hollis D, et al. CALGB 80403/ECOG 1206: a randomized phase II study of three standard chemotherapy regimens (ECF, IC, FOLFOX) plus cetuximab in metastatic esophageal and GE junction cancer. J Clin Oncol. 2010;28(Suppl 15):a4006.

37. Chan JA, Blaszkowsky LS, Enzinger PC, et al. A multicenter phase II trial of single-agent cetuximab in advanced esophageal and gastric adenocarcinoma. Ann Oncol. 2011;22(6):1367-1373.

38. Gold PJ, Goldman B, Iqbal S, et al. Cetuximab as second-line therapy in patients with metastatic esophageal adenocarcinoma: a phase II Southwest Oncology Group Study (S0415). J Thorac Oncol. 2010;5(9):1472-1476. 
39. Tebbutt NC, Sourjina T, Strickland AH, et al. ATTAX2: docetaxel plus cetuximab as second-line treatment for docetaxel-refractory oesophagogastric cancer-final results of a multicentre phase II trial by the AGITG. Proceedings of the GI ASCO; 2008:a87.

40. Stein A, Al-Batran SE, Arnold D, Peinert S, Siewczynski R, Schmoll HJ. Cetuximab with irinotecan as salvage therapy in heavily pretreated patients with metastatic gastric cancer. Proceedings of the GI ASCO; 2007:a47.

41. Rao S, Starling N, Cunningham D, et al. Matuzumab plus epirubicin, cisplatin and capecitabine (ECX) compared with epirubicin, cisplatin and capecitabine alone as first-line treatment in patients with advanced oesophago-gastric cancer: a randomised, multicentre open-label phase II study. Ann Oncol. 2010;21(11):2213-2219.

42. Kim, YH, Sasaki Y, Lee KH. et al. Randomized phase II study of nimotuzumab, an anti-EGFR antibody, plus irinotecan in patients with 5-fluorouracil-based regimen-refractory advanced or recurrent gastric cancer in Korea and Japan: Preliminary results. J Clin Oncol. 2011;29(Suppl 4):a87.

43. Dragovich T, McCoy S, Fenoglio-Preiser CM, et al. Phase II trial of erlotinib in gastrooesophageal junction and gastric adenocarcinomas: SWOG 0127. J Clin Oncol. 2006;249(30):4922-4927.

44. Rojo F, Tabernero J, Albanell J, et al. Pharmacodynamic studies of gefitinib in tumor biopsy specimens from patients with advanced gastric carcinoma. J Clin Oncol. 2006;24(26):4309-4316.

45. Rodriguez CP, Adelstein DJ, Rice TW, et al. A phase II study of perioperative concurrent chemotherapy, gefitinib, and hyperfractionated radiation followed by maintenance gefitinib in locoregionally advanced esophagus and gastroesophageal junction cancer. $J$ Thorac Oncol. 2010;5(2):229-235.

46. Fornaro L, Lucchesi M, Caparello C, et al. Anti-HER agents in gastric cancer: from bench to bedside. Nat Rev Gastroenterol Hepatol. 2011;8(7):369-383.

47. Meza-Junco J, Au HJ, Sawyer MB. Critical appraisal of trastuzumab in treatment of advanced stomach cancer. Cancer Manag Res. 2011;3: $57-64$.

48. Bang $\mathrm{Y}$, Chung $\mathrm{H}, \mathrm{Xu}$ J, et al. Pathological features of advanced gastric cancer (GC): relationship to human epidermal growth factor receptor 2 (HER2) positivity in the global screening programme of the ToGA trial. J Clin Oncol. 2009;27(Suppl 15S):a4556.

49. Gong SJ, Jin CJ, Rha SY, Chung HC. Growth inhibitory effects of trastuzumab and chemotherapeutic drugs in gastric cancer cell lines. Cancer Lett. 2004;214(2):215-224.

50. Cortés-Funes H, Rivera F, Alés I, et al. Phase II of trastuzumab and cisplatin in patients (pts) with advanced gastric cancer (AGC) with HER2/neu overexpression/amplification. J Clin Oncol. 2007; 25(Suppl 18):a4613.

51. Bang YJ, Van Cutsem E, Feyereislova A, et al. Trastuzumab in combination with chemotherapy versus chemotherapy alone for treatment of HER2-positive advanced gastric or gastro-oesophageal junction cancer (ToGA): a phase 3, open-label, randomised controlled trial. Lancet. 2010;376(9742):687-697.

52. Iqbal S, Goldman B, Lenz HJ, Fenoglio-Preiser CM, Blanke CD. S0413: A phase II SWOG study of GW572016 (lapatinib) as first line therapy in patients (pts) with advanced or metastatic gastric cancer. J Clin Oncol. 2007;25(Suppl 18):a4621.

53. Hecht J, Urba SG, Koehler M, et al. Lapatinib monotherapy in recurrent upper gastrointestinal malignancy: phase II efficacy and biomarker analyses. The Proceedings of GI ASCO; 2008:a43.

Biologics: Targets \& Therapy

\section{Publish your work in this journal}

Biologics: Targets \& Therapy is an international, peer-reviewed journal focusing on the patho-physiological rationale for and clinical application of Biologic agents in the management of autoimmune diseases, cancers or other pathologies where a molecular target can be identified. This journal is indexed on PubMed Central, CAS, EMBase, Scopus
54. Lenz H, Zhang W, Kemner AM. Lapatinib 1 capecitabine in advanced gastric cancer: An open-label phase II study of non ERBB2-targeted disease. Ann Oncol. 2010;21(Suppl 8):S817.

55. Roth A, Moehler MH, Mauer M, et al. Lapatinib in combination with $\mathrm{ECF} / \mathrm{X}$ in EGFR1 or HER2-overexpressing first-line metastatic gastric cancer (GC): a phase II randomized placebo controlled trial (EORTC 40071). J Clin Oncol. 2010;28(Suppl 15):a205.

56. Satoh T, Bang Y, Wang J, et al. Interim safety analysis from TYTAN: A phase III Asian study of lapatinib in combination with paclitaxel as second-line therapy in gastric cancer. J Clin Oncol. 2010;28(Suppl 15): a4057.

57. ClinicalTrials.gov. LOGiC - Lapatinib Optimization Study in ErbB2 (HER2) Positive Gastric Cancer: A Phase III Global, Blinded Study Designed to Evaluate Clinical Endpoints and Safety of Chemotherapy Plus Lapatinib. Available at: http://clinicaltrials.gov/ct2/show/ NCT00680901? term $=$ LOGiC\&rank=8.

58. Di Fiore F, Sesboüé R, Michel P, Sabourin JC, Frebourg T. Molecular determinants of anti-EGFR sensitivity and resistance in metastatic colorectal cancer. Br J Cancer. 2010;103(12):1765-1772.

59. Chen J, Huang XF, Katsifis A. Activation of signal pathways and the resistance to anti-EGFR treatment in colorectal cancer. J Cell Biochem. 2010;111(5):1082-1086.

60. Bardelli A, Siena S. Molecular mechanisms of resistance to cetuximab and panitumumab in colorectal cancer. J Clin Oncol. 2010;28(7): 1254-1261.

61. Pinto C, Di Fabio F, Siena S, et al. Phase II study of cetuximab in combination with FOLFIRI in patients with untreated advanced gastric or gastroesophageal junction adenocarcinoma (FOLCETUX study). Ann Oncol. 2007;18(3):510-517.

62. Stella G, Rojas Llimpe FL, Barone C, et al. KRAS and BRAF mutational status as response biomarkers to cetuximab combination therapy in advanced gastric cancer patients. J Clin Oncol. 2009;27(Suppl 15): a15503.

63. Wu WK, Tse TT, Sung JJ, Li ZJ, Yu L, Cho CH. Expression of ErbB receptors and their cognate ligands in gastric and colon cancer cell lines. Anticancer Res. 2009;29(1):229-234.

64. Takiuchi H, Doi T, Muro K, et al. Everolimus in patients with previously treated metastatic gastric cancer: Final results of a multicenter phase II study. The Proceedings of GI ASCO; 2010:a52.

65. Van Cutsem E, Yeh KH, Bang YJ, et al. Phase III trial of everolimus (EVE) in previously treated patients with advanced gastric cancer (AGC): GRANITE-1. J Clin Oncol. 2012;4(Suppl 30):LBA3.

66. Sierra JR, Tsao MS. c-MET as a potential therapeutic target and biomarker in cancer. Ther Adv Med Oncol. 2011;3(Suppl 1):S21-S35.

67. Christoph DC, Schuler M. Polo-like kinase 1 inhibitors in mono- and combination therapies: a new strategy for treating malignancies. Expert Rev Anticancer Ther. 2011;11(7):1115-1130.

68. Ii M, Li H, Adachi Y, et al. The efficacy of IGF-I receptor monoclonal antibody against human gastrointestinal carcinomas is independent of k-ras mutation status. Clin Cancer Res. 2011;17(15):5048-5059.

69. Sankhala KK, Mita MM, Mita AC, Takimoto CH. Heat shock proteins: a potential anticancer target. Curr Drug Targets. 2011;12(14): 2001-2008

70. Marini KD, Payne BJ, Watkins DN, Martelotto LG. Mechanisms of Hedgehog signalling in cancer. Growth Factors. 2011;29(6): 221-234.

and the Elsevier Bibliographic databases. The manuscript management system is completely online and includes a very quick and fair peerreview system, which is all easy to use. Visit http://www.dovepress. com/testimonials.php to read real quotes from published authors.

\section{Dovepress}

